\title{
Ecosophy in the Context of Various Types of Scientific Rationality
}

\author{
Zoya Denikina \\ Department of Sociology \\ FSBEI HE (Financial University affiliated to the \\ Government of the Russian Federation) \\ Moscow, Russia \\ E-mail: zooden@mail.ru
}

\author{
Anatoliy Denikin \\ Department of Sociology \\ FSBEI HE (Financial University affiliated to the \\ Government of the Russian Federation) \\ Moscow, Russia \\ E-mail: andenikin@yandex.ru
}

\begin{abstract}
The article defines the subject and conceptmethodological means of modern ecosophy. The problems of ecological thought are reviewed in the context of the specifics of social systematicity, and the evolution of views on socionatural system is revealed with respect to the existing types of scientific rationality. Notional schemes of classical, non-classical and post-non-classical rationality outline the status of interior and exterior environments of socionatural system. They reveal intrinsic features of ecological socionatural systems and specify the criteria of their ecological performance.
\end{abstract}

Keywords-interior environment; exterior environment; socionatural systems; types of scientific rationality; steady development; ecoobjects; ecosophy

\section{INTRODUCTION}

Ecological thinking issues are directly integrated into the notional specifics of social systematicity, and the evolution of views on the social system and its environment is accordingly defined by paradigm shifts in social knowledge. The main features of classical paradigm of social knowledge are specified in Marxist and Soviet philosophy [Learn more about classical social cognition: 11]. The works of $\mathrm{T}$. Parsons feature further paradigm changes, the author of the most detailed structural and functional theory of social systems uses the principles of non-classical social knowledge. According to Parsons, a social system is never structured in such a way that it becomes radically incompatible with the conditions for its functioning. In order to reflect the balance and stability of a social system by non-classical means, one must combine methodological principles of the theory of social systems with the theory of social action [8]. Russian scientists are performing massive analytic work in order to comprehend non-classical social knowledge [for example, 9].

Globalization processes have a vast effect on modern social knowledge. In post-non-classical view, social reality looks like an intermediate result of the "end of society" [12] as "network" society (M. Castels), as "sociology of mobilities" (J. Urri) [10]. In this epistemological case, it is problematic to describe its structural properties, the integrity of social system, its interactions with the environment. Nevertheless, post-non-classical interdisciplinary knowledge finds methodological resources for synergetics, cybernetics of the second order of $\mathrm{H}$. fon Hoerster, autopoesis of Varela and Maturana, helping to describe interaction between the social system and the environment. Russian science adapts synergetic ideas to the rendering of the development of social systems [See, for example, 2].

Within the context of modern nanotechnical science, the following questions are debated: "what specific kind of evolution are we talking about: biological, social and possibly biosocial; where and who (or what) must "direct" this evolution; what shapes it may take" [1].

\section{ON THE SPECIFICS OF ECOSOPHY}

Modern reflection of ecological problems is associated with the self-definition of ecosophy. The term was introduced by Norwegian philosopher Arne Ness [6], who accentuates emotional-value ties with nature, with other people, with the surrounding natural environment. A more profound definition is provided by Z. Hull, pointing out the ecological flows in the philosophy of nature. The common subject in ecosophical philosophy of nature is "nature and human simultaneously, in their unity, interdependence and interaction, reviewed from the point of their essence, ways of existence and cognition, value and evaluation, moral qualification and the ability to foresee and regulate for the benefit (existence) of human and biosphere..." [3]. The problematic field of ecosophy is so wide, that it can be reconstructed in the notional schemes of the philosophy of nature, philosophy of politics, philosophy of culture, philosophical anthropology, philosophy of physics, e.t.c. The end of the XX century sees the arrival of the interdisciplinary formulation of ecosophical problems, solution of which is inseparably connected with the axiological component, the human lifeworld.

Modern philosophy of science reveals new resources of philosophical and ecological reflection. While in the $\mathrm{XX}$ century ecosophy was directed at human existence, value structures of human reality, currently it is also conjoined with system reality, basic structures of system existence. On the one hand, in the modern philosophy of science, the status of ontology is being transformed, essential ontology is understood "as comprehension of ontological schemes of theoretical activity, ontological status of ideal objects and 
theoretical constructs" [7]. On the other hand, a large role in the bonding of "constructive" and fundamental ontology [See, for example, 5] is played by comprehension of regulatory and methodological meaning of types of scientific rationality. The tendency of the classical science to find empirical referents of ideal constructs is becoming popular, and accordingly, interdisciplinary nature of post-nonclassical science and metalevel of philosophical methodological analysis require a way leading towards synthetic parameters of reality. Naturalism in comprehension of philosophical-scientific notions is increasingly heuristical. Physical objects are also epistemological formations with subject content. Diversity of ontologies also exists within a single discipline and within the borders of the relevant type of scientific rationality. Ecosophy directs disciplinary research into certain conceptual and methodological direction, providing the research with consistency. How is it possible?

One of the directions suggests bonding of various types of scientific rationality. Each of them explains the sociocultural predicament for the used notions, giving real meaning to the conceptual-methodological scheme. Types of scientific rationality conceal the chosen criteria of reality of scientific constructs, they have an overdisciplinary character, and as archetypes, determine the holistic solution of problems. Ecological problem is in essence ontological problem, because there is a threat of extinction of biological species and the whole natural environment. Ecological problem is also in essence a holistic (systematic problem), concerning various ways of interaction between nature and humans. Accordingly, the essence of modern ecosophy is related to the review of certain ontological tenets.

Ecoobjects have system nature regardless of the availability of an intersubjective unity within the scientific community, in this sense, one can speac of an empiric referentiality of system notions. Possible extrapolation of system schemes on subjectivity is a different matter. In modern ecosophy, such schemes are applicable under the scope of view of various ontological consequences, stemming from the types of scientific rationality.

\section{CLASSICAL SOCIAL-PHILOSOPHICAL DISCOURSE AND ECOSOPHY}

In classical social-philosophical scientific discourse, a social system means a system of regularities of its development (for example, Marxist social theory universally adopts this thesis). Ecological cross-section of system reality is revealed with the help of dialectic methodology. The effects of imbalance in the interior system environment are compensated by harmonization of opposites, bolstering the common essence. Presence of opposites in social-natural system ensures its dynamics, diversity and multifariousness. Accordingly, the ecology of interior environment means the condition of essence expansion, characteristic for sustaining essential determinism. Therein, the influence of phenomena parameters is secondary and fragmentary, and the development of social-natural system reveals a minimal number of "converted" forms of existence. The notion of interior environment is organically incorporated into the family of dialectic categories. In this notion, the socialnatural system is presented within the borders of the revelation of essence, with all random, substantive attributes. The result of ecological performance of the interior environment is the achievement of stable development. Stability itself is synonymous with reproduction of regularities, ensuring the linearity of any changes within the system.

The area of interaction of essentially autonomous social systems forms the exterior environment. The ecology of the exterior environment means detection and maintenance of cause-and-effect intersystematic relationships, not destructive towards the essence of the components of the interaction. In ontological schemes of classical philosophy of science, the exterior environment is filled with cause-andeffect relationships rather than functional links. Functional links are bound with latent conditions, variable substantial factors, while cause-and-effect relationships constituate the intersystem environment and determine the logics of intersystem interaction. Thus, the ecology of exterior environment expresses the causality of intersystem interactions, and system ecology - in its steady development, quality maintenance of the carrier of interaction with the environment itself.

\section{NON-CLASSICAL SOCIAL-PHILOSOPHICAL DISCOURSE AND ECOSOPHY}

In non-classical philosophy of science, the social system is defined as a local specific integrity, described by structural-functional methodology. The dynamics of socialnatural system is tied with various ontological coordinates: spatial, temporal and environmental. In different time periods, the system is dominated by different substantial essential parameters. The interior environment of a dynamic natural-social system is an integral part of system reality, consisting of subjective part and system conditions. Only system conditions form an interior environment of functioning. By their nature, they are objectively subjective, objective in space-time sense of system existence and subjective in the context of meaningful actions of system subjects.

The ecology of social-natural system in its non-classical expression expresses the status of relation of the subjective part and its conditions. The system must not pseudo-function in conditions which are alien to it. Stable development as a criterion of ecological performance suggests correct functioning, i.e. maintaining structure and functions after changes of ontological conditions.

The relation of system and exterior environment looks like the problem of search for system adaptation. Forms of adaptation may vary, but the result is always the same: the system neutralizes the determination effect of the environment. The exterior medium of functioning incorporates a wide range of possible transformations of system reality, in any case it remains an ontologically neutral sphere of system existence.

In social system of non-classical type, its teleological capabilities for compensation of undesired environmental 
effects have much importance. The existence of the system and the existence of the medium are intercomplementary concerning ontological equality of the system and the environment. The exterior environment is not megasystematic, non-homogenous, it is multifactorial and diverse. At the same time, the interrelation of the system and exterior environment is modelled not in categories of determination, but in categories of coexistence, such as "functions", "structure", "factors". The notion of stability regarding the exterior environment is non-heuristic. The exterior environment is as it is now, it simply exists, while the processes of adaptation reflect the intrasystem mechanism of functioning.

\section{POST-NON-CLASSICAL SOCIAL-PHILOSOPHICAL DISCOURSE AND ECOSOPHY}

In post-non-classical philosophy of science, the problem of system and environment ecology can be reviewed at least in three different ways. The first option is to address ecological problems within the complex of problems related to self-organization of strongly nonequilibrium systems. The present condition of chaos exit is described by synergetics as a variation of system methodology. One must note, that the location of the border between "order and chaos" is oriented both at constructive human activities, and at subjectless system dynamics. The second option is to view the problem of ecology as a problem of reconstruction and creation of intrasystem factors in order to maintain the systemacity. The difference from the classical approach is that the center of attention is turned not to the stage-by-stage expansion of the essence, but delimitation of the system from the environment, another difference is related to the representation of the mechanism of intrasystem changes.

Such system does not adopt a long-term strategy of changes, while intrasystem interactions are not determined by rigid all-system logics. Systems are imagined not in classical categories of part and element, as static subjects, but within the notions of system processuality, irreversibility of interactions. The existence of such system is complemented by ontological ambiguity caused by the possibility of non-linear spawning of new system fragments.

Autopoetic social-natural system is self-sufficient in the sense that its maintenance does not require the potential of the exterior environment, it rather needs intrasystem reflexion, monitoring of system condition. One must note certain analogies from classical point of view with system ecology. Autopoetic system is certainly "worried" about remaining within the limits of system norm, which is much similar to the struggle of classical system-thing for "purity" of essential parameters.

In the third option, the solution of system and environment ecology problem is represented by constructivist concepts. The problem of ecology is a problem of realization of a system project as a stable ontological structure. Major role is played by the competences of social subjects, their abilities for tactical and strategic forecasting, their social activity stabilizes the "body" of social-natural system. Description of stable conditions uses methodology of social constructivism as a type of system analysis.

All three options demonstrate algorithms of system reductionism. Stable development means exactly simplification of super-complex conditions and further intrasystem changes. The ecology of such systems manifests itself as the arrival of methods of effective management. "It is known that in XIX century, biological science tried to incorporate the idea of evolution into everything and apply the discovered evolutionary mechanisms. It served as the structural support for the mindset of that time. Such role is played nowadays by digital (computer) experimentation and mathematical modelling of processes of self-organization in complex non-linear systems... - this is a kind of reductionism... in the constructive sense of the word" [4].

In all three options, linear description of the relation between the system and the environment is substituted for the ideas of multienvironmental conditions of dynamic integrity.

\section{CONCLUSION}

Nowadays, interdisciplinary character of ecological thinking is beyond doubt. At the basis of ecological mindset, there must be an interdisciplinary methodological principle, which can be called the principle of ontological intricacy, complexity (term borrowed from M. Castels). Accordingly, social-natural system is viewed within the context of integration of natural scientific and social humanitarian knowledge. As definition of social system and its environment complying with the criteria of all types of scientific rationality is hardly achievable, it is more important to determine the intrinsic features of ecological social systems and specify the criteria of their ecological performance. Such system must be seen as human-like and nature-like, having meta-systematic integrity and running in the mode of reproduction, self-regulation or self-organization.

In the age of technological revolution, it is underproductive to speak about distinct division between natural and artificial world. The main criterion of ecological performance of social-natural system is the ontological act of its retention. In classical philosophical reflection, it is fixed by the notion of stable development, and in non-classical correct functioning, in post-non-classical - system selforganization (not only in synergetic interpretation). Thus, in the context of system analysis, ecology is seen as a scientific basis for system crisis recovery, and the very notion of "ecology" is directly tied with characteristics of ontology of social-natural systems.

\section{REFERENCES}

[1] Arshinov the Complexity of post-non-classical practices and the future of converging technologies // post-non-Classical practices: the experience of conceptualization: a Collective monograph / ed. V. I. Arshinova and O. N. Astafieva. - SPb.: Publishing house "Mir", 2012 P. 178.

[2] Budanov V. G. Methodology of synergetics in post-non-classical science and education. Ed. 3-e, DOP. - M.: Book house "LIBROKOM", 2009. - 240 p. 
[3] Hull Z. Ecosophy the philosophy of nature / Philosophy of nature today. - M.: "Canon+" ROOI "Rehabilitation", 2009. P.149.

[4] Knyazeva E. N., Kurdyumov S. P. Synergetics: new universalism or natural philosophy in post-nonclassical science? // Philosophy of nature today. - M.: "Canon+" ROOI "Rehabilitation", 2009. P. 324.

[5] Kozhevnikov N.N., Danilova V.S. Prerequisites for the formation of post-non-classical philosophy and science: monograph. - Yakutsk: NEFU Publishing house. 2014. - P. 260.

[6] Naess F. Defense of Deep Ecology Movement // Environmental Ethics. Vol. 6. 1984 P. 265-270.

[7] Ogurtsov A. P. Philosophy of nature as ontology / / Philosophy of nature today. - M.: "Canon+" ROOI "Rehabilitation", 2009. P.55.

[8] Parsons T. The social system. N.Y., 1964 (Is ted. 1951). P. 207.

[9] Pruginin B. I. Nonclassical epistemology: the view from the classics // Postclassical: philosophy, science, culture: Collective monograph / Ed. Ed. Kiyashenko L. P., and V. S. Stepin. SPb.: Publishing house "Mir", 2009. P. 230-246.

[10] Urri J. Sociologj beyond Societies. Mobilities for the XXIst Centuri. - L., N.Y., P.203.

[11] Vasilkova V. V. Order and chaos in the development of social systems: (Synergetics and theory of social self-organization). $-\mathrm{SPb}$.: LAN Publishing House, 1999. P. 195-197.

[12] Walerstein I. From society to Historical Social Science: Prospects and Obstacles.// The British Jornal of sociologj. - 2000, v. 51, № 1. P. 2536. 